\title{
An Analytical Description of the Packet Train Model for LAN traffic characterization
}

\author{
Maurice Gagnaire, Daniel Kofman and Hayri Korezlioglu \\ Ecole Nationale Supérieure des Télécommunications, \\ 46 rue Barrault, 75634, Paris cedex 13 - FRANCE
}

\begin{abstract}
Resource allocation is a major issue in Broadband ISDN. The more precise the characterization of the flows entering the network is, the easier the resource management becomes. LAN traffic characterization is still an open problem. Different analytical models have been proposed in the literature to describe the packet flow on a LAN. Unfortunately, none of these models correspond to real traffic measurements. In reference to the logarithm histogram of frame interdeparture times from a token ring, Jain and Routhier have proposed an empirical model called the packet train model. This model allows to faithfully describe the statistical behaviour of LAN traffic by means of computer simulations. In the present paper, we give an analytical formulation of Jain and Routhier's empirical model. Our formulation is based on MMPP. We assimilate the frame interdeparture time probability distribution to a phase type probability distribution (PH-distribution). We propose an approach for mapping experimental measurements to the various parameters of our model. The reliability of our approach is confirmed when applied to frame interdeparture times monitored on a token ring.
\end{abstract}

Keywords: Local Area Networks, traffic characterization

\section{INTRODUCTION}

B-ISDNs are based on the ATM technique. This technique allows a gain in the network resource utilization when statistical multiplexing is used. In order to ensure a quality of service to the users connected to B-ISDNs, mechanisms such as Call Admission Control (CAC) and source policing (UPC) have been proposed [1]. The performance evaluation of these three functions (statistical multiplexing, CAC and UPC) requires the characterization of the flows entering B-ISDNs. The more precise the source modeling, the better the prediction of packet interdeparture times, and the easier the prediction of resource requirements . One of the first services to be offered by B-ISDNs is LAN interconnection, hence the necessity for a faithful characterization of LAN traffic behaviour. So far, Interrupted Poisson Processes and Poisson processes with batch arrivals have frequently 
been used for the purpose. Unfortunately, these models do not correspond to real traffic measurements (see for instance [5] or [2]). In reference to the logarithm histogram of measured frame interdeparture times on a token ring, Jain and Routhier have proposed an empirical model called the packet train model (PTM) [3] for LAN traffic characterization. The aim of this paper is to give an analytical formulation of the PTM empirical model.

In Section 2, we underline the lack of reliability of the existing LAN traffic characterizations and describe the principle of the packet train model. In Section 3, we give an analytical formulation of this empirical model. Our formulation is based on Markov modulated Poisson processes (MMPP) (cf. [9]). We propose an approach for mapping experimental measurements of interdeparture times onto the parameters of our analytical model. In Section 4, we obtain an exact formulation of the interdeparture time distribution of our model by means of phase type distributions (PH-distributions) cf. [4]. In order to illustrate the accuracy of the model, we give the characterization of a measured token ring traffic. Finally, our conclusions and our coming investigations following this study are presented in section 5 .

\section{PRINCIPLE OF THE PACKET TRAIN MODEL (PTM)}

Jain and Routhier have monitored frame interdeparture times on a token ring. They observed that the logarithm histogram of these interdeparture times is characterized by three successive linear components (see Figure 1 from [3]). From left to right we will denote the three components I to III. Similar results may be observed on an Ethernet.

Let $f_{I}(t)$ be the probability density function of the random variable $I$ associated to packet interdeparture times on a LAN. The logarithm histogram of $f_{I}(t)$ for a Poisson process is a linear function whereas it is a linear function with a spike at the origin for a Poisson process with batch arrivals. The logarithm histogram of $f_{I}(t)$ for an Interrupted Poisson Process (IPP) is a function with two successive linear components. Thus, none of these three models faithfully describe the statistical behaviour of a LAN traffic.

Jain and Routhier proposed an empirical approach allowing to match the measured three slope behaviour. They distinguished different models called "car" and "packet train" models. Car models are characterized by the independence of frame interdeparture times. The Poisson arrival model is a well-known car model where the frame interdeparture times are exponentially distributed. Packet train models are characterized by the exchange of frame groups between end-user pairs. The PTM LAN traffic model they proposed distinguishes three time scales. At the highest level (macroscopic time scale), data traffic is a succession of "bursts" and "silences". One observes that a burst is itself composed of a succession of "mini-bursts" and "mini-silences" (intermediate time scale). Finally, a mini-burst is composed of several frames of variable length (microscopic time scale). 


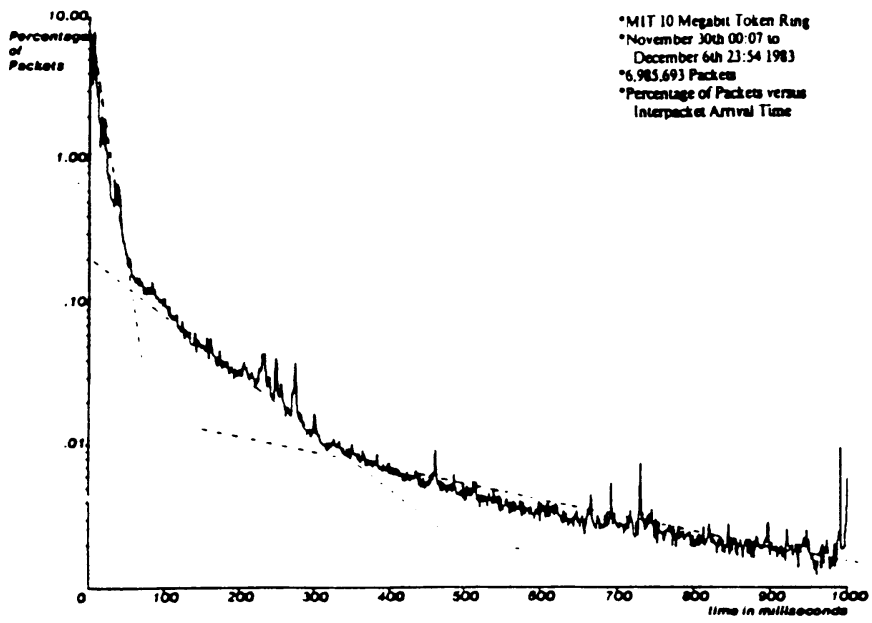

Figure 1. Log histogram of frame interdeparture times for a token ring

Figure 2 allows to interpret Figure 1. Component I (the most frequent interdeparture times) corresponds to the frame interdepartures in a given mini-burst. Component II corresponds, for a given burst, to the interdeparture times between the last frame of the $k$-th mini-burst and the first frame of the $(k+1)$-th mini-burst. Finally, component III corresponds to the interdeparture times between the last frame of the $k$-th burst and the first frame of the $(k+1)$-th burst, which are of course the least frequent. In Figure 2, we have represented the traffic pattern between two end-users $A$ and $B$ where only frames traveling from $A$ towards $B$ are represented.

Although the frames of a same mini-burst are close to each other, they cannot be assimilated to bulk arrivals. The inter-burst time depends on the user's behaviour. The inter-frame time inside a mini-burst and the inter-mini-burst time inside a burst depend on the end-station characteristics (hardware, software and protocols). An extension of the PTM empirical model to a frame relay environment was proposed in [7].

\section{AN ANALYTICAL FORMULATION OF THE PACKET TRAIN MODEL}

In order to describe the three slope behaviour illustrated by Figure 1, we propose to characterize the measured traffic by a 4 state MMPP model with stochastic intensity $\lambda(t)$ such as :

$\lambda(t)=\lambda 1_{\{X(t)=Y(t)=1\}}$ 


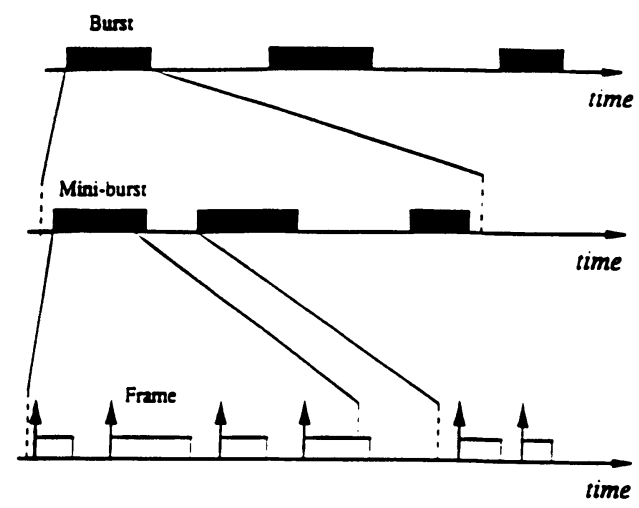

Figure 2. The three time scales of the packet train model

where the processes $X$ and $Y$ are two continuous time Markov chains with values in $\{0,1\}$. In reference to the PTM traffic pattern, these two Markov chains $X$ and $Y$ represent, respectively, the burst and mini-burst scales introduced in the previous section. The four states associated to our MMPP are given by the values of the pair $(X, Y)$ that is $(0,0)$, $(0,1),(1,0)$ and $(1,1)$.

Let us show, by means of formal calculations, how this model correctly characterizes the observed three slope behaviour. We neglect the probability of the nonoccurrence of a frame during a visit of the state $(1,1)$. We also assume that the sojourn time at state $(1,0)$ is negligible compared to the sojourn time of $X$ at its state 1 . Thus, we define three types of frame interdepartures :

- type 1 : They occur while the phase process $(X, Y)$ remains at state $(1,1)$. Such interdepartures occur inside the same mini-burst.

- type 2 : They occur while the phase process $(X, Y)$ goes successively through states $(1,1),(1,0)$ and $(1,1)$. Such interdepartures separate the last frame of a $k$-th mini-burst and the first frame of the $(k+1)$-th mini-burst within the same burst.

- type 3 : Two kinds of events may occur during these interdepartures. Either the phase process $(X, Y)$ jumps from state $(1,1)$ to state $(0,1)$, or it successively goes through states $(1,1),(1,0)$ and $(0,0)$. Such interdepartures separate the last frame of a burst and the first frame of the following burst.

Figure 3 illustrates these three types of interdepartures. 


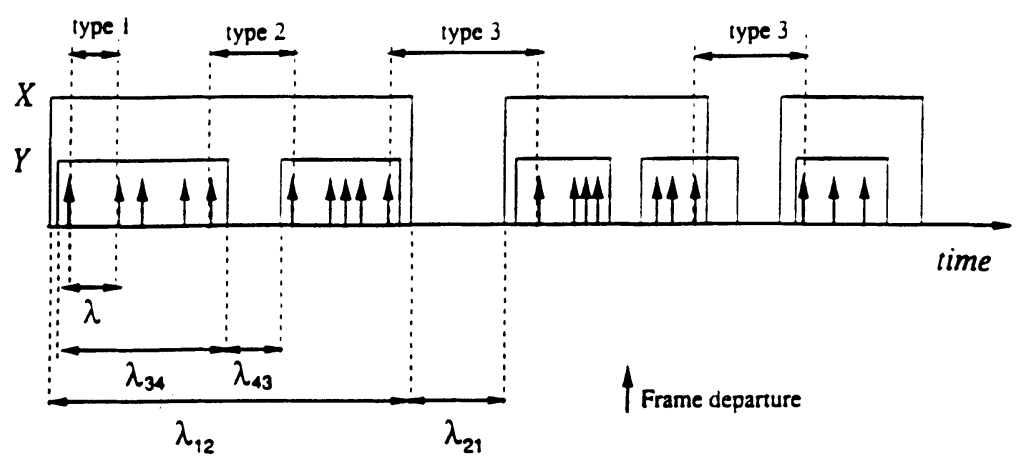

Figure 3.

The three interdeparture types

Let $E$ be a random variable equal to $i=1,2,3$ when the frame interdeparture is of type $i$. Then one has :

$$
\begin{aligned}
& P(I \in[a, a+d x])= \\
& \quad P(I \in[a, a+d x] / E=1) P(E=1)+P(I \in[a, a+d x] / E=2) P(E=2)+ \\
& \quad P(I \in[a, a+d x] / E=3) P(E=3)
\end{aligned}
$$

The following notations will be used:

- $\lambda$ is the arrival rate when the phase distribution is in state $(1,1)$.

- $\lambda_{12}$ is the transition rate for process $X$ from 1 to 0 .

- $\lambda_{21}$ is the transition rate for process $X$ from 0 to 1 .

- $\lambda_{34}$ is the transition rate for process $Y$ from 1 to 0 .

- $\lambda_{43}$ is the transition rate for process $Y$ from 0 to 1 . 
With these notations, we have approximatively:

$$
\begin{aligned}
& P(I \in[a, a+d x] / E=1)=\lambda \exp (-\lambda a) d x \\
& P(I \in[a, a+d x] / E=2)=\lambda_{43} \exp \left(-\lambda_{43} a\right) d x \\
& P(I \in[a, a+d x] / E=3)=\lambda_{21} \exp \left(-\lambda_{21} a\right) d x
\end{aligned}
$$

and

$$
\begin{aligned}
& P(E=1)=\frac{\lambda}{\lambda+\lambda_{34}+\lambda_{12}} \\
& P(E=2)=\frac{\lambda_{34}}{\lambda+\lambda_{34}+\lambda_{12}} \times \frac{\lambda_{43}}{\lambda_{43}+\lambda_{12}} \\
& P(E=3)=\frac{\lambda_{12}}{\lambda+\lambda_{34}+\lambda_{12}}+\frac{\lambda_{34}}{\lambda+\lambda_{34}+\lambda_{12}} \times \frac{\lambda_{12}}{\lambda_{43}+\lambda_{12}}
\end{aligned}
$$

In fact, we have an interdeparture of type 2 if the process $Y$ jumps from state 1 to state 0 and returns to state 1 during a visit of process $X$ to state 1 . This justifies the two factors of the equality (7). We have an interdeparture of type3 whenever, before the next arrival, the process $X$ jumps from state 1 to state 0 . This can occur either directly from the transition $(1,1) \rightarrow(0,1)$ or after the transitions $(1,1) \rightarrow(1,0) \rightarrow(0,0)$. (We recall that we nelect the probability of the nonoccurrence of a frame during a sojourn at state $(1,1))$. This justifies the two terms in equation (8).

In reference to LAN traffic measurements, the parameters characterizing the frame interdeparture process are such that for most values of the variable $a$, two of the three terms in equation (2) are negligible. This explains how our model matches the three slope behaviour observed by Jain and Routhier.

\section{Fitting the PTM analytical formulation in with experimental measurements}

From the approximate calculations of the previous section, we can map the PTM analytical model to the experimental measurements. In fact, from equations (3)-(5) we know that the slopes of the three components are determined by the parametrs $\lambda, \lambda_{43}$ and $\lambda_{21}$. Parameter $\lambda$ also determines the intersection of the first linear component with the vertical axis. In fact, this point is given by $\lambda P(E=1)$ and according to our traffic assumptions $P(E=1)$ is close to 1 . The two other parameters $\lambda_{12}$ and $\lambda_{34}$ determine the points $\beta$ and $\gamma$ given in Figure 5 . The point $\beta$ (respectively $\gamma$ ) corresponds to the intersection between the second (respectively the third) linear part of the log-histogram with the vertical axis. The positions of $\beta$ and $\gamma$ on the vertical axis are respectively given by : 


$$
\begin{aligned}
& \beta=\lambda_{21} P(E=2) \\
& \gamma=\lambda_{43} P(E=3)
\end{aligned}
$$

The characterization of the traffic can be done as follows : we first determine the values of the parameters $\lambda, \lambda_{43}$ and $\lambda_{21}$ by measuring the three slopes. Then equations (7) and (8) allow to compute th values of the parameters $\lambda_{12}$ and $\lambda_{34}$ fixing the position of the linear components II and III.

\section{EXACT COMPUTATION OF THE INTERDEPARTURE DISTRIBUTION}

Our MMPP is a renewal process. In fact, it is known that an MMPP is a renewal process if and only if arrivals occur in a single state of the phase (cf. [9]). The probability distribution of this renewal process is of the phase type. Our approach may be applied to any MMPP for which arrivals occur only in a single state of the phase. In order to derive the analytical expression of $f_{I}(t)$, let us first recall the definition of a phase type distribution.

Consider a $(m+1)$-state Markov chain $A$ with $m$ transient states and one absorbing state. Its infinitesimal generator $Q$ is of the form :

$Q=\left(\begin{array}{cc}T & T^{\circ} \\ \hat{0} & 0\end{array}\right)$

where $T$ is a $m \times m$ matrix with $T_{i, i}<0$ and $T_{i, j}>0$ for $i \neq j$ and such that $T^{-1}$ exists. The term $T^{\circ}$ is a column $m$-vector satisfying $T e+T^{0}=0$ where $e$ is a column vector whose components are all equal to " 1 ". The entry $\hat{0}$ is a row $m$-vector whose components are all equal to " 0 ". Consider a probability vector $\left(\hat{\alpha}, \alpha_{m+1}\right)$, where $\hat{\alpha}$ is a row vector with $m$ components and $\alpha_{m+1}$ is a scalar. Let $A(t)$ be the state of the Markov chain $A$ at instant $t$ when the initial state distribution is $\left(\hat{\alpha}, \alpha_{m+1}\right)$. According to [10], the probability distribution $\Pi_{A}(t)$ of $A(t)$ is given by :

$\Pi_{A}(t)=\left(\hat{\alpha}, \alpha_{m+1}\right) \exp (-Q t)$

The probability that the Markov chain $A$ has not reached its absorbing state at instant $t$ is $\hat{\alpha} \exp (-T t) e$. The absorption time distribution is then given by :

$$
F(t)=1-\hat{\alpha} \exp (-T t) e
$$


Figure 4 represents the evolution of the Markov chain $A$ associated to the interdeparture time of our 4 state MMPP.

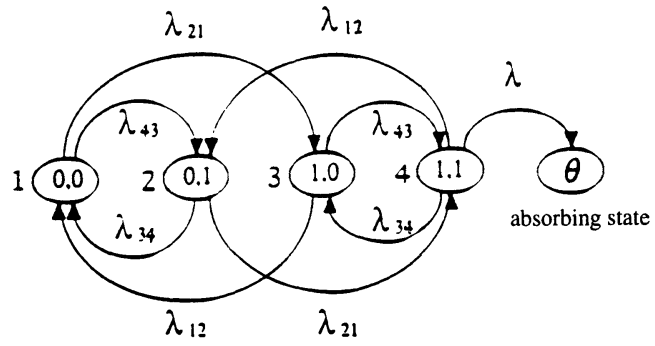

Figure 4. The state diagram of the Markov chain $A$

Let us consider a set $F$ including the 4 transient states of $(X, Y)$ and a 5 -th state $\theta$ denoting the absorbing state. We define on $F$ a Markov chain whose infinitesimal generator is given by :

$Q=\left(\begin{array}{ccccc}-\lambda_{43}-\lambda_{21} & \lambda_{43} & \lambda_{21} & 0 & 0 \\ \lambda_{34} & -\lambda_{34}-\lambda_{21} & 0 & \lambda_{21} & 0 \\ \lambda_{12} & 0 & -\lambda_{12}-\lambda_{43} & \lambda_{43} & 0 \\ 0 & \lambda_{12} & \lambda_{34} & -\lambda_{12}-\lambda_{34}-\lambda & \lambda \\ 0 & 0 & 0 & 0 & 0\end{array}\right)$

where rows and columns are in the order $(0,0),(0,1),(1,0),(1,1)$ and $\theta$. The probability distribution of the interdeparture time $I$ is given by the phase type distribution associated to the absorption time of this Markov chain when the initial state is almost surely state $(1,1)$. By differentiating equation (13), we get the exact analytical expression of $f_{I}(t)$ :

$f_{I}(t)=\hat{\alpha} T e \times \exp (-T t) e$

In order to show the accuracy of our model, we used the approach presented in Section 3 to characterize the traffic measured by Jain and Routhier (see Figure 1) and obtained the following values of the parameters : 
$\lambda=0.083, \lambda_{21}=0.0019, \lambda_{43}=0.015, \lambda_{12}=0.0019$, and $\lambda_{34}=0.015$

Using the formal mathematical language Maple [8], we plotted in Figure 5 the analytical expression of $f_{I}(t)$ given in (15) for the above given values of the parameters.

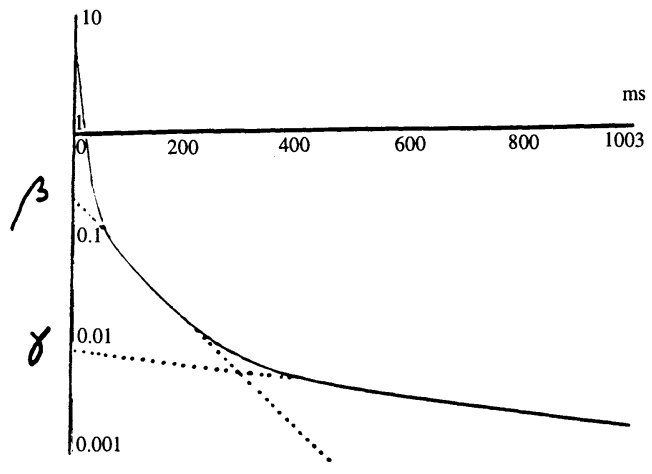

Figure 5. Computed interdeparture times density

It can be seen that our model adequately matches the statistics of Figure 1.

\section{Remark}

The log histogram presented in Figure 1 cannot be directly assimilated to the probability density function $f_{I}(t)$. Jain and Routhier's histogram refers to the percentage of the number $I_{k}$ of interdepartures $I$ such that:

$k \epsilon \leq I<(k+1) \epsilon$

where $\epsilon$ stands for the quantification step. If $N$ is the amount of frames monitored throughout the experiment, then the percentage $P_{I}(x)$ of interdepartures in the interval containing $x$ is given by:

$P_{I}(x)=\sum_{k=1}^{\infty} 1_{\{x \in[k \epsilon,(k+1) \epsilon]\}} \frac{I_{k}}{N}$ 
To assimilate the experimental curve with a probability density function, we have to normalize $P_{I}$, i.e., we have to determine a constant $C$ such that :

$\int_{0}^{\infty} C P_{I}(x) d x=1$

Thus one has $C=\epsilon^{-1}$. In our case we have $\epsilon=1 \mathrm{~ms}$.

\section{CONCLUSION}

The packet train model is an empirical approach used to characterize the statistical behaviour of LAN traffic. In this paper, we have proposed an analytical formulation of this model based on a 4 state MMPP for which the interdeparture time distribution is of the phase type. We have also proposed an approach allowing to match the parameters of this analytical model with the experimental results. We have shown by considering real measurements carried out on a token ring that our analytical model and approach allowing to compute the parameters of the model are quite accurate. In our coming studies, we will extend our model so as to consider the short term correlation between frame interdeparture times. The results obtained in this paper will also be used to study the statistical multiplexing of LAN traffic sources by applying the model presented in [6].

\section{REFERENCES}

1. ITU-T Recommendation I.371. Traffic control and congestion control in B-ISDN, Frozen Issue-Geneva March 1994.

2. R. Gusella, "A Measurement Study of Diskless Workstation Traffic on an Ethernet", IEEE Transactions on communications, September 1990.

3. R. Jain and S. A. Routhier, "Packet Trains - measurements and a new model for computer network traffic", IEEE Journal on Selected Areas in Communications, Vol. SAC-4, N6, September 1986.

4. M. F. Neuts, "A Versatile Markovian Point Process", Journal of Applied Probability, Vol. 16, N4, December 1979.

5. W. E. Leland and D. V. Wilson, "High-Time Resolution Measurement and Analysis of LAN traffic : Implications for LAN interconnection", Proceedings IEEE Infocom'91, Bal Harbour-FL, USA, pp. 1360-1366, April 1991.

6. D. Kofman and H. Korezlioglu, "Loss probabilities and Delay and Jitter Distributions in a Finite Buffer Queue with Heterogeneous Batch Markovian Arrival Processes", Proceedings of GLOBECOM'93, pp 830-834, Houston-TX, USA, Nov. 29 - Dec.3, 1993.

7. M. Gagnaire and T. Uso, "Performance evaluation of Frame Relay based on the packet trains model", Second International IFIP conference on broadband communications, Paris, March 2-4, 1994. 
8. B. W. Char \& Al., "MAPLE Reference Manual", Watcom publications limited, 1988.

9. W. Fisher and K. Meier-Hellstern, The Markov modulated Poisson Process (MMPP) cookbook, Performance Evaluation, Vol. 18 , pp. 149-171, 1992.

10. E. Cinlar, Introduction to stochastic processes, Prentice-Hall, 1975. 